\title{
Value of routine monitoring of bone mineral density after starting bisphosphonate treatment: secondary analysis of trial data
}

\author{
Katy J L Bell, research fellow, ${ }^{1}$ Andrew Hayen, senior lecturer in biostatistics, ${ }^{1}$ Petra Macaskill, associate \\ professor of biostatistics, ${ }^{1}$ Les Irwig, professor of epidemiology, ${ }^{1}$ Jonathan C Craig, professor of clinical \\ epidemiology, ${ }^{1}$ Kristine Ensrud, professor of medicine, ${ }^{2}$ Douglas C Bauer, professor of medicine and \\ epidemiology and biostatistics ${ }^{3}$
}

${ }^{1}$ Screening and Test Evaluation Program, School of Public Health, Edward Ford Building (A27),

University of Sydney, NSW 2006, Australia

${ }^{2}$ University of Minnesota Medical School, Veterans Affairs Medical Center, One Veterans Drive (1110), Minneapolis, MN 55417, USA ${ }^{3}$ Box 0560, 185 Berry Street 5700, University of California, San Francisco, CA 94143-0560, USA Correspondence to: $\mathrm{KJ} \mathrm{L}$ Bell katyb@health.usyd.edu.au

Cite this as: BMJ 2009;338:b2266 doi:10.1136/bmi.b2266

\section{ABSTRACT}

Objective: To assess the value of monitoring response to bisphosphonate treatment by means of measuring bone mineral density.

Design Secondary analysis of trial data using mixed models.

Data source The Fracture Intervention Trial, a randomised controlled trial that compared the effects of alendronate and placebo in 6459 postmenopausal women with low bone mineral density recruited between May 1992 and May 1993. Bone density measurements of hip and spine were obtained at baseline and at one, two, and three years after randomisation.

Main outcome measures Between-person (treatment related) variation and within-person (measurement related) variation in hip and spine bone mineral density. Results The mean effect of three years' treatment with alendronate was to increase hip bone mineral density by $0.030 \mathrm{~g} / \mathrm{cm}^{2}$. There was some between-person variation in the effects of alendronate, but this was small in size compared with within-person variation. Alendronate treatment is estimated to result in increases in hip bone density $\geq 0.019 \mathrm{~g} / \mathrm{cm}^{2}$ in $97.5 \%$ of patients.

Conclusions: Monitoring bone mineral density in postmenopausal women in the first three years after starting treatment with a potent bisphosphonate is unnecessary and may be misleading. Routine monitoring should be avoided in this early period after bisphosphonate treatment is commenced.

\section{INTRODUCTION}

Osteoporosis and associated fragility fractures are major threats to the health of ageing populations worldwide. ${ }^{1}$ Evidence based clinical guidelines provide advice on how to identify and treat individuals at high risk of fracture..$^{2-6}$ Bisphosphonates in particular are important for preventive treatment. What is less certain is how to determine an individual's true response once treatment is started.

Guidelines for treatment of postmenopausal osteoporosis differ in their recommendations for monitoring after starting bisphosphonates. The US National Osteoporosis Foundation and the American Association of Clinical Endocrinologists recommend routine monitoring of bone mineral density within two years of starting treatment. ${ }^{23}$ The UK National Osteoporosis Guidelines Group, US National Institutes of Health, and the Osteoporosis Society of Canada do not make a recommendation either way on monitoring. ${ }^{457}$ The UK guidelines recommend that further research is needed and the North American guidelines recommend that treatment should not be stopped or changed because of a modest observed loss in density.

Bone density monitoring of patients receiving treatment consumes considerable health resources. The availability of bone densitometry varies substantially between countries, with about 10 times as many densitometers per million population in the US as in the UK (35.8 v 3.7 scanners/million population). Although this reflects use of densitometry for case finding as well as for monitoring treatment, it is likely that over half of all measurements done are for monitoring. ${ }^{8}$

Meta-analyses of trials of anti-resorptive agents such as bisphosphonates have found significant association between treatment effects on bone mineral density and fracture risk, ${ }^{9-11}$ with the relation seeming to be stronger for non-vertebral factures ${ }^{11}$ than vertebral fractures. ${ }^{910}$ Although this suggests that bone density may be considered as an intermediate outcome for monitoring treatment effects at a population level, more evidence is needed before we can decide whether it should be used for monitoring treatment effects at an individual level.

In this paper we investigate the effects of alendronate on bone density at the individual level. We compare the variability of bone density for patients receiving placebo with that for patients taking treatment, using mixed models ${ }^{12}$ to make inferences about whether the effect of treatment varies among individuals or is uniform. If the treatment effect did not vary between individuals we could infer that monitoring individuals' response to treatment is unnecessary as the effect of 
the bisphosphonate on the individual could be predicted before treatment is started. A finding that treatment effect varied between individuals may imply that monitoring individuals' response to treatment is necessary if the variation is great enough to be clinically relevant. This would occur if a substantial proportion of patients failed to meet a treatment threshold-for example, if the treatment effects ranged from no effect (or even reduction in bone density) up to a large gain in bone density (see Bell et al ${ }^{13}$ for a more detailed discussion on when variation in treatment effects is likely to be clinically relevant). We used data from a large randomised trial to determine whether routine monitoring of bone mineral density is warranted after starting alendronate treatment.

\section{METHODS}

\section{Study design and population}

We analysed data from the Fracture Intervention Trial (FIT), a randomised trial that compared the effects of alendronate with placebo in 6459 postmenopausal women with low bone mineral density $\left(\leq 0.68 \mathrm{~g} / \mathrm{cm}^{2}\right.$ at baseline) ${ }^{14}$ The trial had two arms - the vertebral fracture arm, which included 2027 women who had vertebral fractures identified on radiographs at baseline,$^{15}$ and the clinical fracture arm, which included 4432 women without baseline vertebral fractures. ${ }^{16}$ Patients for both arms were recruited between May 1992 and May 1993. Data from both arms are included in this paper, with an adjustment made for the arm a particular patient was in by including a term to represent trial arm in the models. Analysis was by intention to treat, and all subjects were included regardless of compliance with study treatment or fracture outcomes.

Patients were randomly allocated to daily alendronate or placebo. Alendronate dose was initially $5 \mathrm{mg} /$ day for two years but was increased to $10 \mathrm{mg}$ /day at the second annual visit because other trials suggested that $10 \mathrm{mg} /$ day had greater effects on bone mineral density. Women in each treatment group who had dietary calcium intakes $<1000 \mathrm{mg} /$ day at baseline $(82 \%$ of participants) were asked to take a daily supplement containing $500 \mathrm{mg}$ of elemental calcium and $250 \mathrm{IU}$ of vitamin D.

\section{Monitoring measurements}

Bone mineral density was measured at the hip and posterior-anterior (PA) spine on all participants with Hologic QDR 2000 densitometers at baseline and at yearly intervals after randomisation. Serial measurements were made with the same machine at each clinical centre. Quality control measures have been described previously. ${ }^{14}$ We used bone mineral density data from baseline and the first three years after randomisation to evaluate response to treatment.

\section{Statistical analysis}

Mixed models are a powerful method for analysing data from longitudinal studies, in which there are multiple measurements on each subject. ${ }^{17}{ }^{18}$ This approach allows explicit modelling of the within-person and between-person variation in the outcome, while taking into account the correlation between measurements taken on the same individual. In this type of analysis, predictive factors may be fitted to have the same effect for everyone, in which case they are said to be fixed effects (these effects are equivalent to the estimated effects in ordinary least squares regression models). Alternatively, the predictive factors may be fitted to have effects that differ between individuals, in which case they are said to have random effects (these effects are not allowed for in ordinary least squares regression models). These alternative models can be compared using likelihood ratio tests to determine which model provides the best fit for the data. If there is no variation in the treatment effect between patients then we would expect the mixed models to find that treatment has a fixed effect and no random effects. Conversely, if there is variation in the treatment effects between patients then we would expect the mixed models to find that treatment has random effects.

We fitted a series of mixed models using bone mineral density measurements over three years. We used the likelihood ratio test to compare models where treatment effect was the same for everyone (treatment had a fixed effect) with models where treatment effect differed between individuals (treatment had random effects). (For a more detailed explanation of model fitting, see appendix 1 on bmj.com and Bell et $\left.\mathrm{al}^{12}\right)$.

For fixed effects we estimated the mean effect applicable to all patients, whereas for random effects we estimated the mean and standard deviation of effects across patients. For random effects we also estimated a 95\% distribution of treatment effects, an interval in which the treatment effect for $95 \%$ of the study population should lie (mean value $+/-(1.96 \times$ standard deviation)).

As the primary analysis we used hip bone mineral density measurements alone (or univariate response models). The hip is the best site for predicting risk of hip fracture and osteoporotic fractures overall ${ }^{19}{ }^{1}$ However many clinicians use both hip and spine bone density measurements to monitor treatment effects. Hip and spine bone mineral density measurements made at the same time on the same individual are correlated. ${ }^{20}$ As an extension to the analysis outlined above, we also fitted models that considered both hip and spine bone mineral density simultaneously, while allowing for their correlation (or bivariate response models).

Analysis was done using MLwiN software package (Centre for Multilevel Modelling, University of Bristol) with models fitted using iterative generalised least squares.

\section{RESULTS}

The results of the primary mixed models analysis (where hip bone mineral density was the outcome) are shown in figures 1 and 2 (the full statistical results are presented in appendix 2 on bmj.com). Fig 1 shows the mean bone mineral density trajectories for 
alendronate and placebo groups from year 1 to year 3 after starting treatment. These represent the mean effects (based on both the fixed and random effects) found in the final mixed model. The mean effect of time in the placebo group was a decrease of $0.004 \mathrm{~g} /$ $\mathrm{cm}^{2}$ per year $(\mathrm{P}<0.001)$, shown by the downward slope for the placebo mean trajectory in fig 1 . The mean effect of one year's treatment with alendronate (relative to placebo) was an increase of $0.013 \mathrm{~g} / \mathrm{cm}^{2}$ $(\mathrm{P}<0.001)$, which is shown in fig 1 by the difference in the mean trajectories for alendronate and placebo at year 1 (the intercept). The mean effect of time in the alendronate group (relative to the placebo group) was an increase in bone mineral density of $0.0085 \mathrm{~g} / \mathrm{cm}^{2}$ per year $(\mathrm{P}<0.001)$, shown by the diverging trajectories (difference in slopes) of the two treatment groups in fig 1 . There was no evidence that treatment effect was modified by baseline bone density $(\mathrm{P}=0.65)$, age $(\mathrm{P}=0.65)$, body mass index $(\mathrm{P}=1.0)$, general health $(\mathrm{P}=0.11)$, or trial arm $(\mathrm{P}=0.32)$.

Fig 2 shows the variance between individuals at the three time points and represents the variances of the random effects of the final mixed model (excluding residual variances). We first consider the placebo variance trajectory. There was considerable variation between patients in the placebo group at one year $(\mathrm{P}<0.001)$, which is shown in fig 2 by the variance of the placebo group at year 1 . In addition, there was variation between individuals in how their bone mineral density changed over time $(\mathrm{P}<0.001)$, shown by the increasing variance in both groups over the three years.

There was also considerable within-person variation over time (not shown in the figures). The within-person variation was significantly lower $(\mathrm{P}<0.001)$ in the group receiving alendronate (standard deviation $0.012 \mathrm{~g} / \mathrm{cm}^{2}$ ) compared with the placebo group (SD $\left.0.014 \mathrm{~g} / \mathrm{cm}^{2}\right)$. Bland-Altman plots $^{21}$ showed that within-person variation did not depend on the individual's mean level of bone mineral density (data not shown).

After allowing for the statistically significant random effects of background sources of variation, there

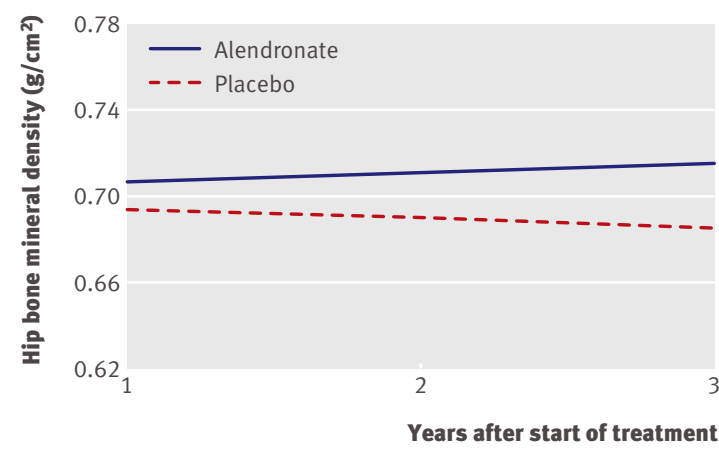

Fig 1| Mean bone mineral density over time for 6459 postmenopausal women with low bone mineral density treated with alendronate or placebo. (As this is a randomised trial, at year 0 the mean bone mineral density is similar for the alendronate and placebo groups) remained strong evidence of variation between individuals in the effects of alendronate on hip bone mineral density $(\mathrm{P}=0.008)$, shown in fig 2 by the difference in the variance trajectories for alendronate and placebo at year 1. Although the variation in treatment effects was statistically significant, it was small in size with a standard deviation of $0.006 \mathrm{~g} / \mathrm{cm}^{2}$, which is about half the standard deviation of within-person variation on treatment. A comparison of variances was even more marked, with the variance of between-person variation in treatment effects being only about one tenth the variance of background within-person variation. The 95\% distribution for the between-person effects of one year's treatment on hip bone density did not overlap zero, ranging from an increase in hip bone mineral density of $0.002 \mathrm{~g} / \mathrm{cm}^{2}$ (2.5th centile) to an increase of $0.024 \mathrm{~g} / \mathrm{cm}^{2}$ (97.5th centile).

There was no strong evidence of additional betweenperson variation in treatment effects after one year $(\mathrm{P}=0.07)$, shown in fig 2 by the parallel variance trajectories of the two treatment groups. After three years, the mean cumulative treatment effect was $0.030 \mathrm{~g} / \mathrm{cm}^{2}$ $\left(0.013 \mathrm{~g} / \mathrm{cm}^{2}+\left(2 \times 0.0085 \mathrm{~g} / \mathrm{cm}^{2}\right)\right)$. The standard deviation of between-person variation in cumulative treatment effect was $0.006 \mathrm{~g} / \mathrm{cm}^{2}$, the same as the variation in treatment effect after one year. The $95 \%$ distribution for the effects of three years' treatment ranged from an increase in bone mineral density of $0.019 \mathrm{~g} / \mathrm{cm}^{2}(2.5$ th centile) to an increase of $0.041 \mathrm{~g} /$ $\mathrm{cm}^{2}$ (97.5th centile).

The bivariate response analysis (where both hip and spine bone mineral density were considered together) yielded similar conclusions to the univariate response analysis presented above. We found strong evidence of between-person variation in the effects of treatment $(\mathrm{P}<0.001)$, but again the absolute size of this variation was small (standard deviation of between-person variation in treatment effect was $0.006 \mathrm{~g} / \mathrm{cm}^{2}$ for hip and $0.007 \mathrm{~g} / \mathrm{cm}^{2}$ for spine). In the bivariate response analysis the $95 \%$ distributions for the effect of one and three years' treatment with alendronate on hip bone mineral density were the same as the univariate analysis. The $95 \%$ distribution for the effects of one year's

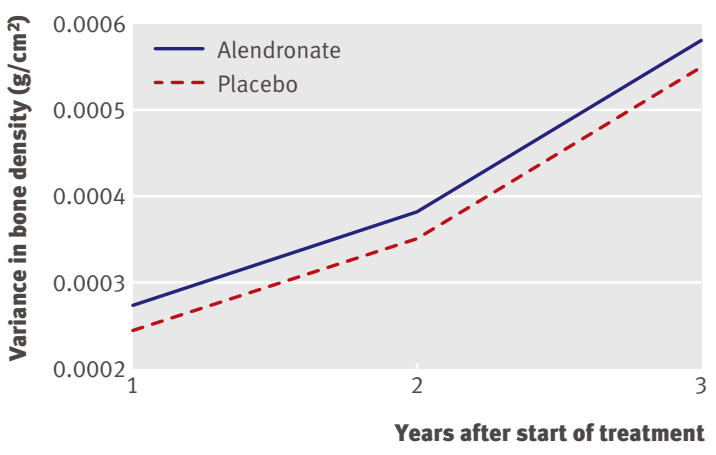

Fig 2 | Between-person variances in bone mineral density over time for 6459 postmenopausal women with low bone mineral density treated with alendronate or placebo. (As this is a randomised trial, at year 0 the between-person variance is similar for the alendronate and placebo groups) 
treatment on spine bone mineral density did not overlap zero and ranged from an increase of $0.015 \mathrm{~g} / \mathrm{cm}^{2}$ (2.5th centile) to an increase of $0.042 \mathrm{~g} / \mathrm{cm}^{2}$ (97.5th centile). At the end of three years, the $95 \%$ distribution for the effects of treatment on spine bone mineral density ranged from an increase of $0.037 \mathrm{~g} / \mathrm{cm}^{2}(2.5$ th centile) to an increase of $0.064 \mathrm{~g} / \mathrm{cm}^{2}$ (97.5th centile). The correlation between the effects of treatment on hip bone mineral density and spine bone mineral density over the three year time period for an individual was 0.90 .

\section{DISCUSSION}

In this large placebo controlled trial of alendronate treatment of postmenopausal women with low bone density and with serial bone mineral density measurements, we found that three years of treatment resulted in an average increase in hip bone mineral density of $0.030 \mathrm{~g} / \mathrm{cm}^{2}$ against a background average decrease in hip bone mineral density of $0.012 \mathrm{~g} / \mathrm{cm}^{2}$ with placebo. We found small between-person differences in the effects of alendronate on bone mineral density. These differences were small compared with background variation in bone mineral density measurements within patients. Moreover, treatment seemed beneficial for the vast majority of patients. Three years of treatment with alendronate resulted in increases in hip bone mineral density $\geq 0.019 \mathrm{~g} / \mathrm{cm}^{2}$ for $97.5 \%$ of patients. Increases in bone density of at least this size are likely to be considered sufficient to continue therapy in most clinical populations. This means that, even though we found the effects of alendronate to vary between individuals, the size of this variation is not clinically relevant and monitoring individual response is not needed. The high correlation between the effects of treatment on hip bone mineral density and spine bone mineral density $(r=0.90)$ confirms previous findings ${ }^{19}$ that there is minimal gain from taking measurement at two sites rather than one. Our findings suggest that bone density monitoring is not warranted at either hip or spine skeletal sites.

The large within-person variation in bone density we found in this study is likely to be a best case scenario, as bone density measurements made in clinical trials probably have considerably less within-person variation than measurements made in clinical practice. Within-person variation that is at least as large as that we have estimated indicates that any true differences in treatment effects in a clinical setting are likely to be hidden. This provides further rationale to not routinely monitor bone density response to oral bisphosphonate treatment.

Clinicians may cite other reasons for monitoring besides estimating the intended effects of treatment. One common reason given is to assess adherence to treatment. ${ }^{2}$ However, the large background withinperson variation for bone mineral density means that monitoring is unlikely to give reliable information about whether the drugs were taken as prescribed. Patient's self reports on structured interview often correlate better with pill count (a reference standard for adherence measures) than do intermediate outcomes (such as blood pressure measurement of patients taking antihypertensive drugs).$^{22}$ In addition, most problems with adherence to osteoporosis treatments occur within three months of starting treatment, ${ }^{23}$ much earlier than the first monitoring for response at one year, providing further argument against routine bone density monitoring for enhancing adherence to treatment. Non-adherence is best detected by direct interview ${ }^{22}$ and best increased by making patients active participants in their treatment. ${ }^{24}$

Other potent oral bisphosphonates such as risedronate and ibandronate operate by a similar mechanism to alendronate. ${ }^{25} \mathrm{It}$ is reasonable, therefore, to generalise our results on the effects of three years of alendronate treatment to the effects of other potent oral bisphosphonates of roughly equivalent dose in postmenopausal women. Further work is needed to generalise beyond this group of drugs-for example, analyses of data from trials evaluating intravenous bisphosphonates and non-bisphosphonates (such as oestrogen, oestrogen agonist/antagonists, parathyroid hormone, and strontium ranelate) are needed to determine the value of monitoring with these treatments. Where data are available on individual patients, mixed models can be used to estimate the variation in treatment effects. ${ }^{12}$ Where only summary data are available, an alternative method may be used that has more restrictive assumptions ${ }^{13}$ (see appendix 3 on bmj.com for an example of this method). Future trialists could directly report on the variation between people in treatment effects, in addition to the average treatment effect that is often reported at present. In this way not only would trials inform clinical decisions on which treatment to choose but also when response monitoring may be helpful (when there is clinically relevant variation between people in the treatment effects).

Our findings support a recommendation against routine monitoring in the first three years after potent bisphosphonate therapy is started. Other work with data from the Fracture Intervention Trial also supports not monitoring bone density in the first few years of alendronate treatment. Women observed to have no net change in bone mineral density after four years of treatment were found to have a high probability of responding to treatment. ${ }^{26}$ Reductions in fracture risk with alendronate treatment were shown for women who were observed to lose bone density during treatment. ${ }^{27}$ Failure to recognise this measurement variation may lead clinicians to make inappropriate changes to treatment or to delay taking action when they should intervene. ${ }^{2328}$

In summary, bone density increases sufficient to continue therapy unaltered occurred in virtually all osteoporotic women treated with a potent oral bisphosphonate for three years. Monitoring bone mineral density in this early period after starting treatment is unnecessary, and, because of the potential to mislead, it is best avoided. 


\section{WHAT IS ALREADY KNOWN ON THIS TOPIC}

Many clinicians monitor bone mineral density to estimate a patient's response to bisphosphonate therapy for osteoporosis, but there is no evidence that this practice is of clinical benefit

Monitoring bone mineral density consumes considerable health resources

The observed response to bisphosphonate therapy may not reflect the true response because of measurement variability

\section{WHAT THIS STUDY ADDS}

Between-person variation in the effects of alendronate on bone mineral density is small compared to within-person variation and is not clinically relevant

Monitoring bone mineral density in postmenopausal women after starting a potent oral bisphosphonate is unnecessary and, because of the potential to mislead, is best avoided

Contributors: KJLB conceived the study, did the analysis and wrote the paper. AH helped with the analysis and helped write the paper. JCC and KE helped write the paper. PM and LI helped supervise the analysis and write the paper. DCB provided the raw data and assisted with manuscript revisions. KJLB is guarantor for the study.

Funding: This study was funded by the Australian National Health and Medical Research Council (Public Health Postgraduate Research Scholarship No 457212 and Program Grant No 402764 ). The Fracture Intervention Trial was sponsored by Merck Research Laboratories. The sponsors did not influence the design and conduct of the study; collection, management, analysis, and interpretation of data; preparation and approval of the manuscript; or decision to submit the article for publication. All of the researchers involved in this project are independent of the funding bodies.

Competing interests: DCB has received honorariums from Merck, Procte \& Gamble, Amgen, Roche Diagnostics, and Novartis; and research support from Procter \& Gamble, Novartis, and Amgen.

Ethical approval: Ethical approval was not required for this study.

1 Johnell O, Kanis JA. An estimate of the worldwide prevalence and disability associated with osteoporotic fractures. Osteopor Int 2006;17:1726-33.

2 AACE Osteoporosis Task Force. American Association of Clinical Endocrinologists medical guidelines for clinical practice for the prevention and treatment of postmenopausal osteoporosis: 2001 edition, with selected updates for 2003. Endocr Pract 2003;9:544-64

3 National Osteoporosis Foundation. Clinician's guide to prevention and treatment of osteoporosis. Washington DC: NOF, 2008. (Available from: www.nof.org)

$4 \mathrm{NIH}$ Consensus Development Panel on Osteoporosis Prevention Diagnosis and Therapy. Osteoporosis prevention, diagnosis, and therapy. JAMA 2001;285:785-95.

5 Brown JP, Josse RG, Scientific Advisory Council of the Osteoporosis Society of Canada. 2002 clinical practice guidelines for the diagnosis and management of osteoporosis in Canada. CMAJ 2002;167(10 suppl):S1-34

6 Writing Group of the Bone and Tooth Society of Great Britain and Royal College of Physicians. Osteoporosis. Clinical guidelines for prevention and treatment. Update on pharmacological interventions and an algorithm for management. 2000. www.rcplondon.ac.uk/pubs/wp/wp_osteo_update.htm.
7 National Osteoporosis Guidelines Group (NOGG). Osteoporosis clinical guideline for prevention and treatment. 2008 (www. iofbonehealth.org/download/osteofound/filemanager/ policy_advocacy/EU-consultation-panel-meetings/overview-uknational-osteoporosis-guidelines-group-08.pdf)

8 Kanis JA, Johnell O. Requirements for DXA for the management of osteoporosis in Europe. Osteopor Int 2005;16:229-38.

9 Wasnich RD, Miller PD. Antifracture efficacy of antiresorptive agents are related to changes in bone density. J Clin Endocrinol Metab 2000;85:231-6.

10 Cummings S, Karpf D, Harris F, Genant H, Ensrud K, LaCroix A, et al. Improvement in spine bone density and reduction in risk of vertebral fractures during treatment with antiresorptive drugs. Am J Med 2002;112:281-9.

11 Hochberg MC, Greenspan S, Wasnich RD, Miller P, Thompson DE, Ross PD. Changes in bone density and turnover explain the reductions in incidence of nonvertebral fractures that occur during treatment with antiresorptive agents. J Clin Endocrinol Metab 2002;87:1586-92.

12 Bell KJL, Hayen A, Macaskill P, Craig JC, Neal B, Irwig L. Mixed model showed no need for initial response monitoring after starting antihypertensive therapy. J Clin Epidemiol 2009;62:650-9.

13 Bell KJL, Irwig L, Craig JC, Macaskill P. Use of randomised trials to decide when to monitor response to new treatment. $B M$ 2008;336:361-5.

14 Black DM, Nevitt MC, Cauley J, Karpf D, Cummings SR. Design of the fracture intervention trial. Osteopor Int 1993;3(suppl 3):S29-39.

15 Black D, Cummings S, Karpf D, Cauley J, Thompson D, Nevitt M, et al. Randomised trial of effect of alendronate on risk of fracture in women with existing vertebral fractures. Lancet 1996;348:1535-41.

16 Cummings S, Black D, Thompson D, Applegate W, Barrett-Connor E, Musliner T, et al. Effect of alendronate on risk of fracture in women with low bone density but without vertebral fractures. JAMA 1998;280:2077-82.

17 Goldstein H, Browne W, Rabash J. Multilevel modelling of medical data. Stat Med 2002;21:3291-315

18 Singer J, Willet J. Doing data analysis with the multilevel model for change. In: Applied longitudinal data analysis. New York: Oxford University Press, 2003:75-137.

19 Leslie WD, Lix LM, Tsang JF, Caetano PA. Single-site vs multisite bone density measurement for fracture prediction. Arch Int Med 2007;167:1641-7.

20 Lu Y, Genant HK, Shepherd J, Zhao S, Mathur A, Fuerst TP, et al. Classification of osteoporosis based on bone mineral densities. Bone Miner Res 2001;16:901-10.

21 Bland JM, Altman DG. Measurement error. BMJ 1996;313:744

22 Haynes RB, Taylor DW, Sackett DL, Gibson ES, Bernholz CD, Mukherjee J. Can simple clinical measurements detect patient compliance. Hypertension 1980;2:757-64.

23 Cummings S, Palermo L, Browner W, Marcus R, Wallace R, Pearson J, et al. Monitoring osteoporosis therapy with bone densitometry: misleading changes and regression to the mean. JAMA 2000;283:1318-21.

24 Haynes RB, Sackett DL, Taylor DW. How to detect and manage low patient compliance in chronic illness. Geriatrics 1980;35:91-7.

25 Roelofs AJ, Thompson K, Gordon S, Rogers MJ. Molecular mechanisms of bisphosphonates: current status. Clin Cancer Res 2006;12(20 suppl):6222-30s.

26 Pavlic M, Brand B, Cummings S. Estimating probability of nonresponse to treatment using mixture distributions. Stat Med 2001;20:1739-53.

27 Chapurlat RD, Palermo L, Ramsay P, Cummings S. Risk of fracture among women who lose bone density during treatment with alendronate. The Fracture Intervention Trial. Osteoporos Int 2005; 16:842-8.

28 Morton V, Torgenson D. Regression to the mean: treatment effect without the intervention. J Eval Clin Pract 2004;11:59.

Accepted: 5 February 2009 